\title{
Self and Chemical Diffusion in Liquid Al-Ag
}

\author{
M. Engelhardt ${ }^{1, a}$, A. Meyer ${ }^{1, b}$, F. Yang ${ }^{1, c}$, G. G. Simeoni ${ }^{2, d}$ and F. Kargl ${ }^{1, e}$ \\ 1 Institut für Materialphysik im Weltraum, Deutsches Zentrum für Luft- und Raumfahrt, 51170 Köln, \\ Germany \\ ${ }^{2}$ Heinz Maier-Leibnitz Zentrum (MLZ) and Physics Department, Technische Universität München, \\ D-85748 Garching, Germany \\ amarc.engelhardt@dlr.de, bandreas.meyer@dlr.de, ${ }^{\mathrm{c}}$ fan.yang@dlr.de, ${ }^{\mathrm{d}} \mathrm{ggsimeoni@outlook.com,}$ \\ eflorian.kargl@dlr.de
}

Keywords: self-diffusion, chemical-diffusion, X-ray radiography, quasi-elastic neutron scattering, Darken relation, liquid metal alloys

\begin{abstract}
Self- and chemical diffusion coefficients are reported for molten Al-Ag on the Al-rich side of the phase diagram for Ag concentrations of up to 45at\% and for pure liquid Ag. Temperature dependent Ag self-diffusion coefficients were obtained using quasi-elastic neutron scattering. Chemical diffusion coefficients were measured in situ by means of X-ray radiography of a longcapillary furnace. A detailed error analysis for the long-capillary experiments is reported. It is shown that perturbing effects can be detected and that accurate chemical diffusion coefficients can be measured with high precision. It is demonstrated based on Al-Ag20at\% that the Darken equation appears to be valid for this system with a thermodynamic factor lower than unity. Furthermore, in $\mathrm{Al}$-Ag it appears that Ag self-diffusion for small Ag concentrations is faster than Al-self diffusion in liquid Al. This contrasts with observations made for other Al-based melts like Al-Ni and Al-Cu.
\end{abstract}

\section{Introduction}

$\mathrm{Al}-\mathrm{Ag}$ is one of the binary base systems of the ternary Ag-Al-Cu alloys. Ag-Al-Cu and its binary constituent systems have been extensively studied in the past years from a thermodynamic point of view [1,2]. This provided a solid basis for studying pattern formation in ternary eutectics both experimentally [3-5] as well as by means of simulations [6]. The underlying phase-field models were thereby further developed in order to obtain a deeper understanding of eutectic pattern formation in this system.

For phase-field modelling of solidification chemical diffusion coefficients of the liquid are an important input parameter. However, only few diffusion data are available for liquid Al-based alloys [7,8] and in particular they are still unknown for the binary constituent systems Al-Ag and Ag-Cu of the ternary eutectic alloy $\mathrm{Ag}_{17.6} \mathrm{Al}_{68.6} \mathrm{Cu}_{13.8}$. In general, the few data available for liquid metal and alloy systems normally show a relatively large scatter. More accurate experiments have so far been limited to lower melting point materials [9-12]. The absence of data can be explained by perturbing effects leading to larger systematic errors in the diffusion coefficients obtained by classical methods like long-capillary experiments with post-mortem sample analysis. In this context, convective flow for instance was identified as a major source of error.

In recent years, it has been shown that quasi-elastic neutron scattering (QENS) is a powerful method to measure accurate self-diffusion coefficients in liquids with high precision [13]. The technique accesses the relevant information on microscopic time- and length-scales. Thereby it is capable of providing benchmark data not perturbed by convective flow.

However, for chemical diffusion coefficient measurements, QENS would only be suited for very few alloys. In order to measure chemical diffusion with QENS the material has to fulfill particular scattering conditions. For instance for a binary alloy one of the constituting elements has to have a positive and the other one a negative scattering length. This is typically only obtained if suitable isotopes are available. The experiment is further restricted to a particular sample composition for 
which the mean scattering length vanishes. In addition, both elements shall not have any incoherent cross section. Therefore, chemical diffusion studies inevitably have to use a classical approach, namely, processing macroscopically-sized capillary samples and determining concentration profiles. Very recently it was shown by monitoring these experiments in real-time using radiography techniques (X-rays [9] and neutrons [10]) that perturbing effects can be detected and that the data can be analyzed such that the chemical diffusion coefficient is not perturbed by these effects [11].

In the present paper self-diffusion coefficients of Ag in pure liquid Ag and an Al-Ag alloy have been determined employing QENS using a time-of-flight spectrometer and processing of the samples in a classical high temperature furnace. Chemical diffusion coefficients on a large range of liquid Al-Ag alloys were obtained combining X-ray radiography in the laboratory with the longcapillary furnace method.

It is shown that Ag self-diffusion follows an Arrhenius temperature dependence in the investigated temperature interval above the liquidus temperature for AlAg20at\% and for pure liquid Ag. Chemical diffusion coefficients initially decrease with increasing Ag content to a roughly constant value. Ag self-diffusion appears to be faster than Al self-diffusion at least at low $\mathrm{Ag}$ concentrations. A comparison of chemical and self-diffusion coefficients using the thermodynamic data tabulated in literature [1] indicates the Darken relation to be valid within the experimental error bars. Cross correlations appear to be negligible.

\section{Sample Preparation and Experimental Details}

For the experiments high-purity $\mathrm{Al}$ (Chempur spherules 99.99\%) and Ag (Chempur spherules 99.9\%) have been used. For the QENS experiments an AlAg20at\% alloy has been prepared by melting of the appropriate amount of the raw materials in a cold crucible under high purity Argon atmosphere. The obtained alloy ingot was inductively melted under vacuum in a centrifugal casting device (Lifumat-Met-3,3-Vac-Titan, Linn High Term) and cast into $1 \mathrm{~mm}$ thin plates. The plates were polished to a thickness of $500 \mu \mathrm{m}$, cut to appropriate dimensions, and inspected by X-ray radiography for shrinkage holes and inhomogeneities. For pure Ag only the centrifugal casting step was carried out. By rotation casting the oxygen skin of the prepared ingot was typically left in the melting container and fresh material was injected in the mold. The obtained defect-free and homogeneous plates were enclosed in a flat plate Nb-sample holder providing a flat-plate sample geometry of $36 \mathrm{~mm}$ width and $50 \mathrm{~mm}$ height. The filled Nb-container was sealed by electron-beam welding under ultra-high vacuum atmosphere.

For the chemical diffusion experiments the Al-Ag alloys were prepared by arc melting in a suction casting device (MAM-1, Edmund Bühler GmbH) under high purity Argon atmosphere. Melting was carried out three-times whereby the sample was flipped over in order to obtain a homogeneous alloy with Ag fully dissolved. Subsequently the raw material was remelted in the suction casting device and cast into a $1.49 \mathrm{~mm}$ diameter capillary crucible obtaining a rod of about $40 \mathrm{~mm}$ length. The rods were radiographed for the detection of shrinkage holes. Shrinkage-hole free rods were cut to appropriate length for the interdiffusion experiments. In the X-ray radiography interdiffusion experiments seven different sample pairs with the compositions AlAg4at\%/Al, AlAg7.5at\%/AlAg12.5at\%, AlAg17.9at\%/AlAg22.9at\%, AlAg15.4at\%/AlAg25.4at\%, AlAg27.5at\%/AlAg32.5at\%, AlAg35.5at\%/AlAg40.5at\%, and AlAg40at\%/AlAg45at\% were processed.

The QENS experiments were carried out on the time-of-flight neutron spectrometer TOFTOF at the Heinz Meyer-Leibniz Zentrum (HLZ) in Garching nearby Munich. The samples were processed in a high-temperature vacuum furnace with Niobium resistance heating elements. The flat-plate samples were held under a $\sim 27^{\circ}$ angle to the incoming neutron beam. Repeatedly accurate sample alignment was obtained by a laser fixed to the sample holding stick. The scattering experiment was therefore carried out with the sample in reflection geometry. The reason for this is the relatively high absorption coefficient of Ag of about 63barn at $1.8 \AA$ neutron wavelength. The experiments were carried out with $7 \AA$ incoming neutrons. The experiment took advantage of the recent upgrade of the neutron-guide system, due to installation of a non-linearly focusing component (Adaptive 

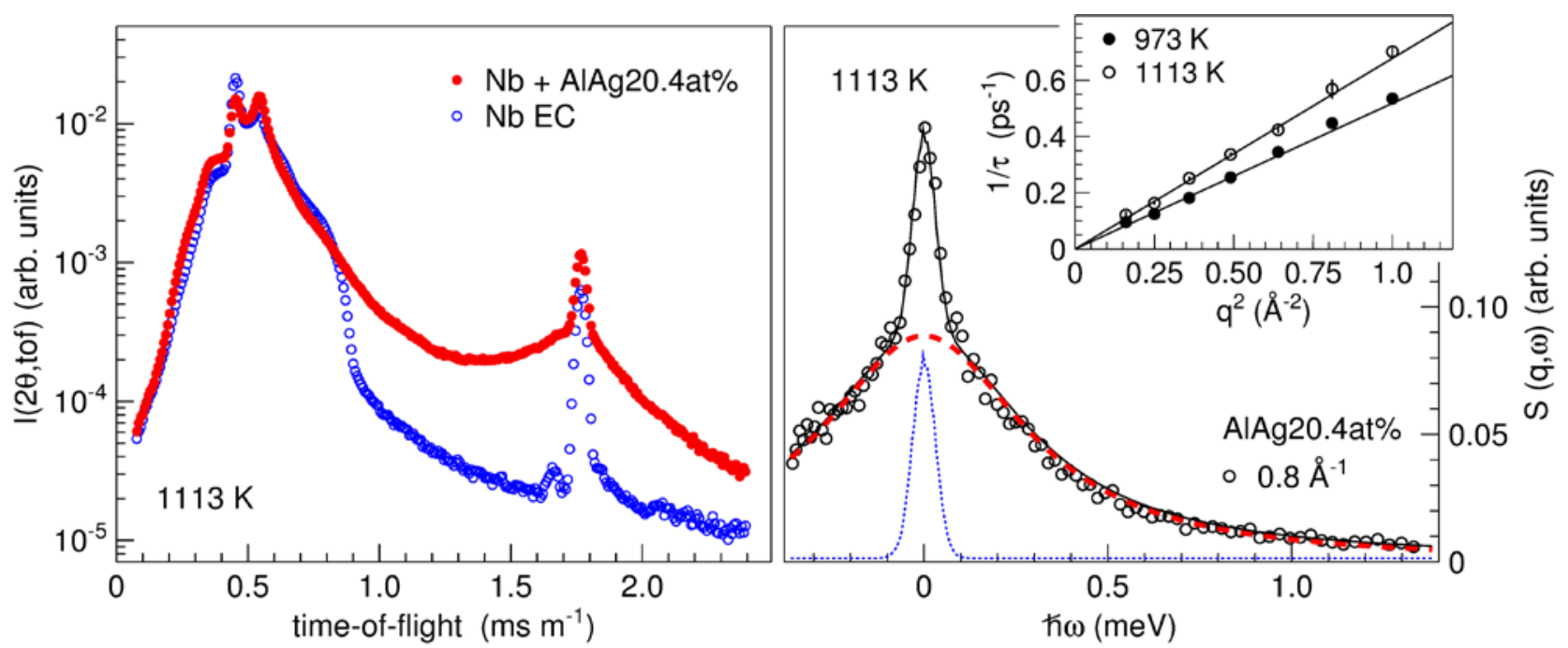

Figure 1 Left: Scattered intensity as function of time of time-of-flight. The data are shown averaged over scattering angles in the range of $30^{\circ}$ to $120^{\circ}$ used in the subsequent data analysis. The scattering of the empty Nb flat-plate sample holder (open symbols) is shown in comparison to the scattering of the AlAg20.4at\% sample and the sample holder (closed symbols). Right: Dynamic structure factor $S(q, \omega)$ at $q=0.8 \AA^{-1}$. Measured data (circles), quasielastic scattering contribution (dashed red line), remaining elastic scattering contribution of the $\mathrm{Nb}$ sample holder (dotted blue line), and total fit to the data (solid black line). Inset: Inverse relaxation time as function of the squared wavevector for two different temperatures. The slopes of the fitted lines correspond to the self-diffusion coefficients.

Optics technology). As a result, the data acquisition benefitted from almost doubled beam intensity and a signal-to-background ratio enhanced by one order of magnitude. Moreover, the focusing geometry strongly reduced the instrumental background at small scattering angles and improved the symmetry of the elastic line [14]. The sample-holder scattering contribution at the elastic line is about a factor five lower than the one of the sample and at least an order of magnitude lower in the region of the quasi-elastic scattering [cf. Fig. 1 (left)]. The data analysis followed standard procedures outlined in Ref. /13/. The measured intensities [cf. Fig. 1 (left)] are converted into the scattering law $S(q, \omega)$ or by Fourier transform into the density correlation function $S(q, t)$. Both, $S(q, \omega)$ and $S(q, t)$ are fitted by suitable equations with the aim to determine the q-dependent halfwidth at half maximum $\Gamma$ or the characteristic decay time $\tau$, respectively. In the present case, different to the standard analysis procedure of fitting only a quasi-elastic scattering contribution represented by a Lorentzian line to $S(q, \omega)$ also an additional elastic contribution for the scattering of the container has to be included [cf. Fig. 1 (right)]. In $S(q, t)$ this elastic contribution results in a constant background to which the exponential function decays (not shown). The diffusion coefficient is determined by using the following relation: $\Gamma \propto 1 / \tau=D q^{2}$ [cf. inset Fig. 1 (right)].

The interdiffusion experiments were carried out using a microfocus X-ray source (XT9160-TED Viscom AG) with a tungsten film on Al transmission target. The entire Bremsstrahlungs-spectrum was used in the experiments. The X-ray source was operated at an acceleration voltage of $150 \mathrm{kV}$ and an electron beam current of $80 \mu \mathrm{A}$. A Shad-o-Box $2048 \mathrm{EV}$ (RadIcon) detector was used for transmitted X-ray beam detection. The detector was operated at a recording speed of $1 \mathrm{fps}$ and provided a native pixel size of $48 \mu \mathrm{m}$. The samples have been placed between the source and the detector such that a magnification of 1.5-2 was achieved resulting in an effective per pixel size of around $32 \mu \mathrm{m}$ to $24 \mu \mathrm{m}$, respectively. The samples were processed in a long-capillary furnace under vacuum already used in earlier studies [9,12]. The image data analysis equally followed procedures outlined in Ref. /9/ and /12/, also discussed below in further detail. The pressure close to the sample was in the range of $10^{-3} \mathrm{mbar}$. 


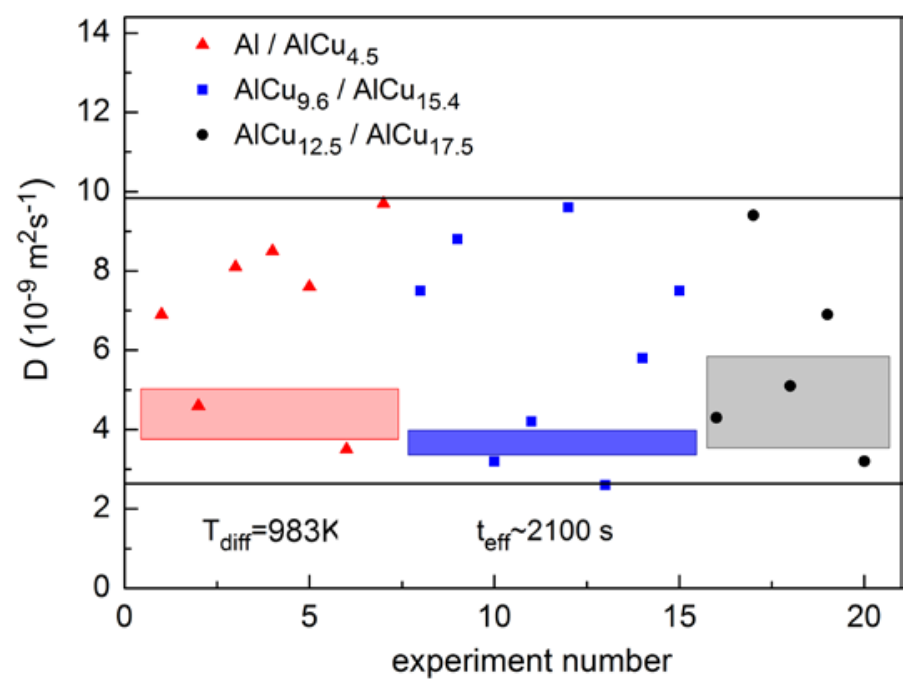

Figure 2 Overview of interdiffusion coefficients in Al-Cu alloys using ex situ long-capillary experiments. The ex situ data deviate by between $-50 \%$ to $+250 \%$ of the real values as determined by in situ measurements using X-ray radiography (cf. colored boxes). A large scatter in the experimental data can be observed for experiments carried out on the same interdiffusion pair under the same experimental conditions (cf. Ref. /15/).

\section{Interdiffusion: Post-mortem vs. In Situ}

In the past decades diffusion coefficients of pure liquid metals and alloys have been experimentally investigated (e.g. pure liquids [16], liquid alloys [17] and even multicomponent Albased alloys [18]) using different methods and experimental facilities. An excellent overview for Al-based melts is given by $\mathrm{Du}$ et al. in Ref. [8]. There, it is for instance shown that the long capillary (LC) method is the most widely used technique for the determination of diffusion coefficients in metal alloys systems. In the past, experiments were carried out with post-mortem analysis of the solidified sample subjected to a full heating, annealing and cooling cycle [16-18]. However, more recently the accuracy of the method was largely improved by coupling the LC method with radiography for in situ observation of the chemical diffusion in metal alloys [9-12].

Using the LC method for chemical diffusion measurements, thin, cylindrical samples of different compositions are pairwise brought into contact with each other in a chemically inert environment. The diffusion couple is melted, annealed at a defined temperature and subsequently quenched. The concentration profile after experiment completion (post-mortem) is analyzed and a diffusion coefficient is derived by fitting a suitable solution of Fick's $2^{\text {nd }}$ law of diffusion to the data. For sample pairs made of the very same alloy, the self-diffusion coefficient can be derived by marking individual atoms by isotopes. However, the classical long capillary method poses several disadvantages, negatively affecting the accuracy of the determined diffusion coefficients. Main causes for the scattering of the post-mortem data are the missing process control, perturbations of the diffusion profile by melting and solidification as well as buoyancy and Marangoni convection. Convective perturbations are e. g. discussed in Ref. [19]. Already minor gradients in density and temperature within the diffusion sample lead to additional convective flows. Also an often necessary coordinate transformation of the solidified sample to its molten state, requiring the knowledge of its density and of its thermal expansion coefficient, impairs the accuracy of the analysis. All the above noted effects affect the measurement and are in case of in situ process control by X-ray radiography reflected in a strong scattering of the diffusion coefficients as exemplarily shown in Fig. 2 for Al-Cu alloys.

Using X-ray radiography the temporal evolution of the concentration profile is followed in realtime. This is achieved by recording the transmitted X-ray intensity on a flat-panel detector with a typical time resolution of 1s. The recorded intensity stored in grey-values can be converted to solute concentration within the alloy sample assuming constant density (to a good approximation valid for small concentration changes) and a parallel X-ray beam. A direct correlation between grey-value and solute concentration can be established using Beer's law for the transmitted intensity $I$ [20]:

$$
I=I_{0} e^{-\mu d}
$$



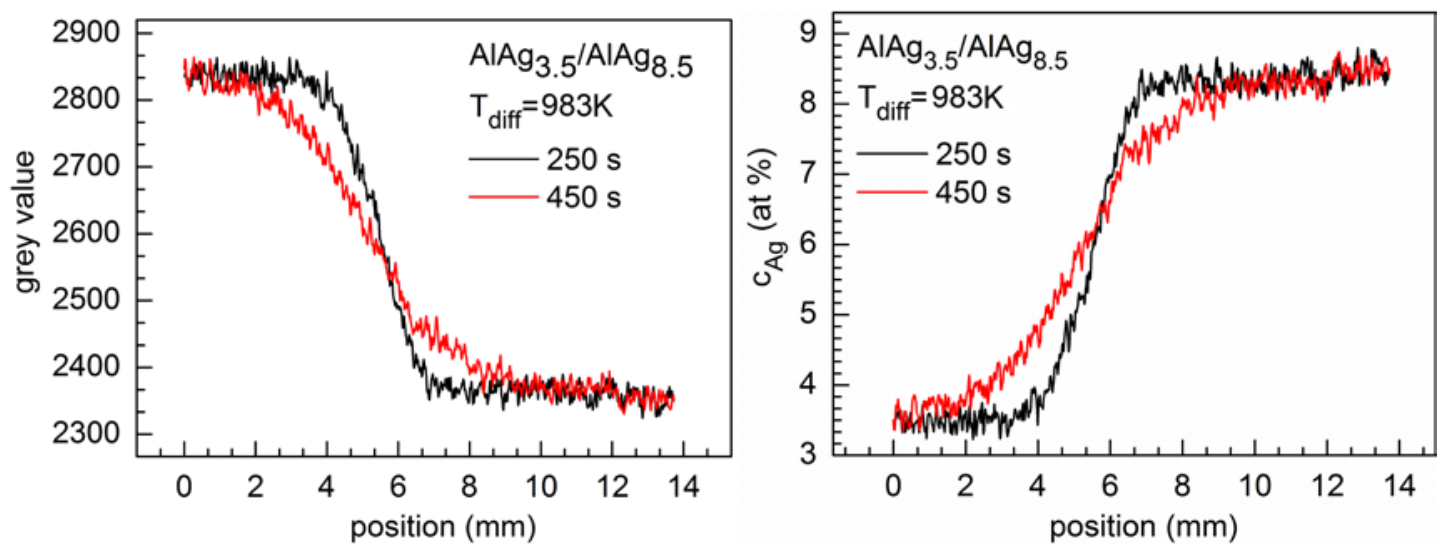

Figure $3 \mathrm{Ag}$ concentration profiles for a AlAg3.5at\%/AlAg8.5at\% diffusion couple. Left: Measured grey values. Right: Corresponding converted Ag concentrations. Data are shown at two different diffusion times.

Herein $I_{0}$ denotes the incoming beam intensity, $\mu$ the absorption coefficient and $d$ the thickness. The assumptions stated above are only approximations. However, it was shown that the error caused by the differences in path length between a parallel beam and the cone-shaped beam of the x-ray source used in this study are negligible [21]. For small concentration variations used in the experiments the density variations over the whole sample length is less than $10 \%$. This was shown to be considerably smaller than the measurement error of the x-ray radiography method [22].

For a binary system like Al-Ag the absorption coefficient can be described as a sum of the individual absorption coefficients of the elements weighted with the respective element concentration $c_{i}$ reading:

$$
\mu=c_{A l} \mu_{A l}+c_{A g} \mu_{A g}=\left(1-c_{A g}\right) \mu_{A l}+c_{A g} \mu_{A g}
$$

Combining Eq. 1 and Eq. 2 the transmitted beam intensities for the two alloys constituting the diffusion couple - each having a different Ag concentration $c_{i}^{A g}$ with $i=1,2$ - read:

$$
\begin{aligned}
& I_{1}=I_{0} \exp \left\{-\left[c_{1}^{A g} \mu_{A g}+\left(1-c_{1}^{A g}\right) \mu_{A l}\right] d\right\} \\
& I_{2}=I_{0} \exp \left\{-\left[c_{2}^{A g} \mu_{A g}+\left(1-c_{2}^{A g}\right) \mu_{A l}\right] d\right\}
\end{aligned}
$$

Given that the initial Ag concentrations $c_{i}$ are known, the time- and position dependent axial Ag concentration $c(x, t)$ is obtained by

$$
c(x, t)=\frac{\ln I(x, t)-\ln I_{1}}{\ln I_{2}-\ln I_{1}} .
$$

$I(x, t)$ are the experimentally determined transmitted intensities axially along the capillary sample. To determine $c(x, t)$ the intensities are treated as directly proportional to the recorded grey values. To illustrate the conversion procedure detailed in Eq. 4, data are shown for AlAg6at\% in Fig. 3. This figure also shows that a sufficiently high contrast is reached in the X-ray radiography experiments, which clearly distinguishes the two alloys concentrations and enables one to derive the temporal evolution of Ag concentration with high precision. To calibrate the effective pixel size by converting pixel number in a real length in units of millimeters, a $1 \mathrm{~mm}$ diameter spherical and chemically inert X-ray absorbing sample was used inside of the capillary furnace. The recorded $c(x, t)$ profiles are described with a solution of Fick's $2^{\text {nd }}$ law reading:

$$
c(x, t)=\frac{c_{2}+c_{1}}{2}+\frac{c_{2}-c_{1}}{2} \operatorname{erf}\left[\frac{x-x_{0}}{\sqrt{4 D_{I D} t}}\right] .
$$



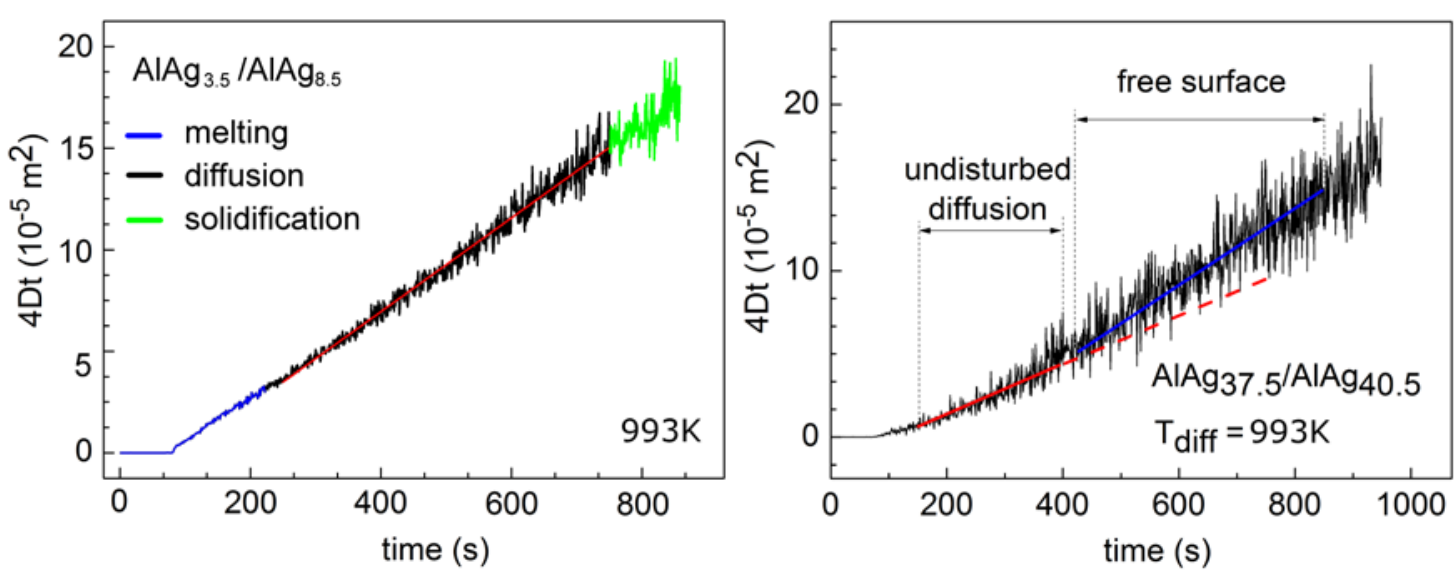

Figure 4 Left: Mean-squared diffusion length as function of time. $L^{2}=4 D t$ is shown for melting, the isothermal annealing (diffusion) and solidification of an AlAg3.5at\%/AlAg8.5\% diffusion couple. The straight line represents a fit to the diffusion phase, where constant temperature conditions prevail. Its slope is proportional to the interdiffusion coefficient. Right: $L^{2}$ shown for a AlAg37.5at\%/AlAg40.5at\% diffusion couple. Two straight line fits are indicated covering different time intervals. In the first interval diffusion was not perturbed, whereas in the second interval diffusion was enhanced by convective flow due to free surfaces formed by a gas bubble.

Here the upper index $A g$ was omitted for the initial concentrations $c_{i}$. The interdiffusion coefficient is denoted by $D_{I D}$, erf denotes the Gaussian error function, and $x_{0}$ is the position in the capillary at which the composition is changed by $50 \%$ between the initial alloy concentrations. Fitting Eq. 5 to the data [e. g. the data depicted in Fig. 3 (right)] two free parameters are used, namely $x_{0}$ and the squared diffusion length $L^{2}=4 D_{I D} t$. In order to obtain the interdiffusion coefficient, $L^{2}$ is plotted as a function of time $t$. This is in Fig. 4 (left) exemplarily shown for an AlAg6at\% diffusion couple. Three different regimes are shown in the figure. A first increase in $L^{2}$ is observed when the sample is melting. Here, temperature conditions are not yet stable and therefore the slope of the measured data curve changes with time. Once stable temperature conditions are established, the slope is constant as indicated by the linear fit to the data (cf. part of the curve denoted by diffusion). Finally the sample is cooled down (solidification). Here the slope changes again since diffusion takes place in a semi-solid sample before the sample is fully solidified. Fig. 4 (right) shows a profile for an AlAg39at\% diffusion couple where the diffusion phase was initially unaffected by convective flow and at later stages of the experiment convective perturbations occurred due to free surfaces formed by a gas bubble. As indicated by an increase in the slope of the fit curves, the interdiffusion coefficient is increased by convective flow to an effective diffusion coefficient larger than the real diffusion coefficient. However, the data can still be fitted by a straight line in good agreement with earlier observations for Al-Cu samples reported in Ref. /11/. As described in this reference only the in-depth analysis of the solute concentration laterally in different parts of the capillary or observations of changes in the capillary images enables to distinguish convective flow from purely diffusive conditions.

In order to obtain accurate interdiffusion coefficients the fits to $L^{2}$ are only carried out for experiment times at which stable temperature conditions prevail. This is defined as a variation of furnace temperature of less than $1 \mathrm{~K}$ over the duration of the experiment. This constant temperature regime is usually reached about $150-200$ s after first diffusion is started.

\section{Results and Discussions}

Results of the QENS experiments are shown in Fig. 5 (left) together with results of the interdiffusion experiments at a temperature of $983 \mathrm{~K}$ as a function of the Ag concentration. Fig. 5 (left) focuses on the range of Ag concentrations up to 45at\% Ag content. The self-diffusion coefficient of pure liquid Ag is shown for comparison. The blue squares in the figure indicate 

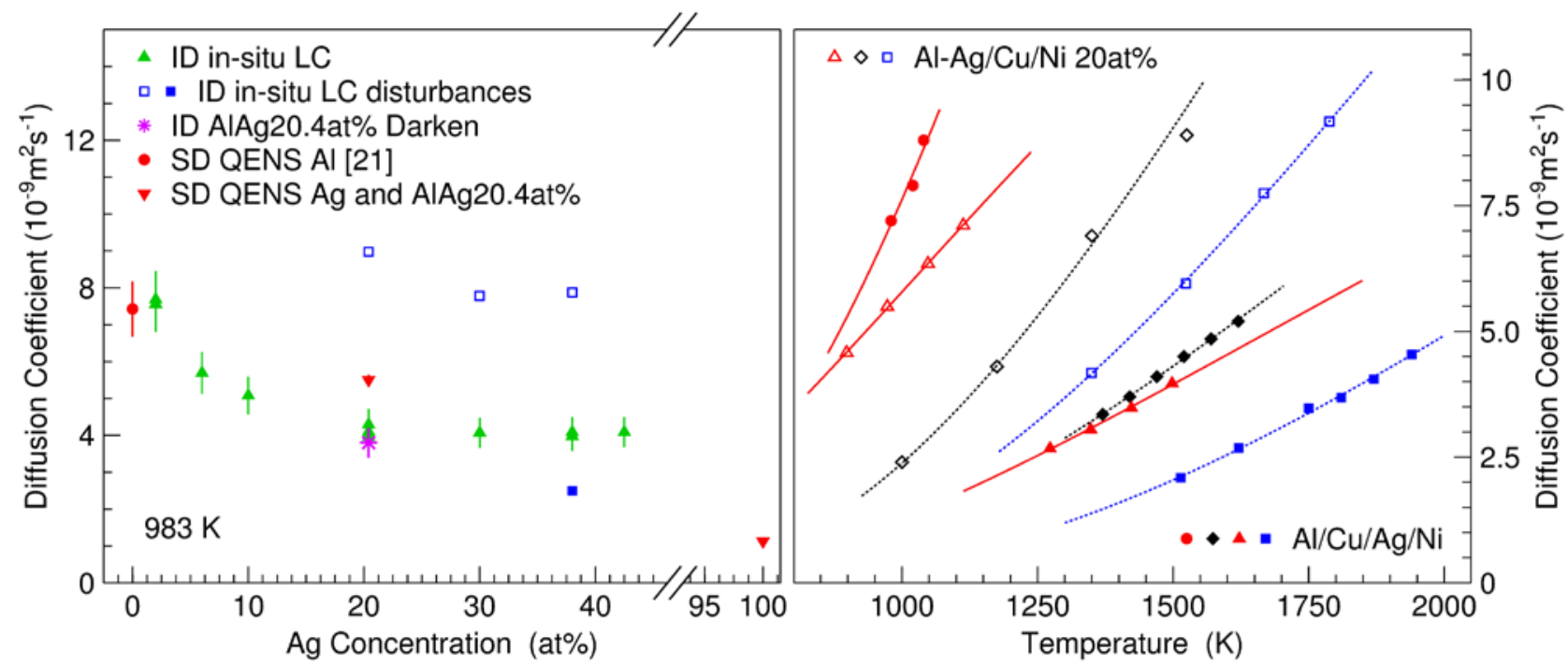

Figure 5 Left: Interdiffusion coefficients as function of $\mathrm{Ag}$ concentration measured at $710^{\circ} \mathrm{C}$ (green up-facing triangles and blue squares), self-diffusion coefficients (red disc and down-facing triangles) and calculated interdiffusion coefficient (purple star). Right: Temperature dependent selfdiffusion coefficients: i) full symbols $\mathrm{Al}$ (discs) [23], $\mathrm{Cu}$ (diamonds) [28], Ag (triangles), and $\mathrm{Ni}$ (squares) [29]; ii) open symbols of Ag in AlAg20.4at\% (triangles), $\mathrm{Cu}$ in $\mathrm{AlCu} 20 \mathrm{at} \%$ (diamonds) [9], and Ni in AlNi20at\% (squares) [25]. Lines represent Arrhenius fits to the data.

measurements perturbed by convective flow (open squares) and by an oxide layer (closed square). They are compared with the unperturbed measurements indicated by the green up-facing triangles. Whereas the data at 30at\% and 38at\% Ag content have been enhanced by gas bubble formation leading to the generation of free surfaces, the data at $20.4 \mathrm{at} \% \mathrm{Ag}$ were enhanced by strong temperature fluctuations.

For the unperturbed measurements the following is observed: Between 20at\% and 42.5at\% Ag content the interdiffusion coefficient appears to be constant at $983 \mathrm{~K}$. Towards lower concentration the interdiffusion coefficient increases. For 2at\% Ag content it almost doubles compared with the data at larger Ag content. For AlAg20.4at\% interdiffusion is slower than Ag self-diffusion. Ag selfdiffusion is faster in AlAg20.4at\% than Ag self-diffusion in pure liquid Ag comparing the QENS data. This compares well with earlier observations for liquid Al-Ni alloys where Ni self-diffusion was reported to decrease with increasing Ni content [24]. Comparing the AlAg20.4at\% with data reported for AlNi20at\% [25] and AlCu20at\% [9] the interdiffusion coefficient is lower than the solute self-diffusion coefficient for $\mathrm{Al}-\mathrm{Ag}$ whereas it is enhanced for $\mathrm{Al}-\mathrm{Ni}$ and $\mathrm{Al}-\mathrm{Cu}$. For $\mathrm{Al}-\mathrm{Cu}$ this is even the case when considering that the interdiffusion data presented in Ref. [9] have been influenced by convective flow as discussed in Ref. [11].

In order to explain this behavior, the interdiffusion coefficient for AlAg20.4at\% is calculated based on the Darken equation [26]. The calculated value is depicted by the purple star in Fig. 5 (left). The Darken equation correlates self- and interdiffusion in binary systems taking into account thermodynamic driving forces but neglecting cross-correlations. The Darken equation reads:

$$
D_{I D}=\left(c_{A l} D_{A g}^{S}+c_{A g} D_{A l}^{S}\right) \Phi
$$

The sum in brackets describes the kinetic part, which is the sum of the element specific selfdiffusion coefficients weighted with the concentrations of the respective other element. $\Phi$ denotes the thermodynamic factor. The thermodynamic factor is the $2^{\text {nd }}$ derivative of the molar Gibbs energy $G$ with respect to concentration. $G$ is given by the sum of the molar Gibbs energy of the pure elements, an ideal mixing term, and an excess Gibbs energy term due to mixing. The excess term is conventionally described by an approximation using Redlich-Kister polynomials (cf. Ref. [1]). For the binary system Al-Ag discussed here, Redlich-Kister polynomials up to the fourth order are 


\begin{tabular}{lcc|lcc}
\hline $\begin{array}{l}\mathrm{T} \\
(\mathrm{K})\end{array}$ & $\begin{array}{c}\mathrm{D}_{\mathrm{Ag}} \mathrm{AlAg} 20.4 \mathrm{at} \% \\
\left(\times 10^{-9} \mathrm{~m}^{2} \mathrm{~s}^{-1}\right)\end{array}$ & $\begin{array}{c}\mathrm{D}_{\mathrm{Ag}} \mathrm{Ag} \\
\left(\times 10^{-9} \mathrm{~m}^{2} \mathrm{~s}^{-1}\right)\end{array}$ & Sample & $\begin{array}{c}\mathrm{D}_{0} \\
\left(\times 10^{-9} \mathrm{~m}^{2} \mathrm{~s}^{-1}\right)\end{array}$ & $\begin{array}{c}\mathrm{E}_{\mathrm{A}} \\
(\mathrm{meV} / \text { atom })\end{array}$ \\
\hline 898 & $4.58 \pm 0.46$ & & $\mathrm{Ag}$ & $37 \pm 3$ & $288 \pm 10$ \\
973 & $5.49 \pm 0.55$ & & $\mathrm{Al}^{[23]}$ & $194 \pm 100$ & $280 \pm 70$ \\
1048 & $6.35 \pm 0.63$ & & $\mathrm{Cu}^{[28]}$ & $58.7 \pm 3$ & $337 \pm 5$ \\
1113 & $7.11 \pm 0.71$ & & $\mathrm{Ni}^{[29]}$ & $77 \pm 8$ & $470 \pm 30$ \\
1273 & & $2.67 \pm 0.27$ & AlAg20.4at\% & $45 \pm 1$ & $176 \pm 6$ \\
1348 & & $3.05 \pm 0.30$ & AlCu20at\% ${ }^{[9]}$ & $131 \pm 11$ & $345 \pm 10$ \\
1423 & & $3.48 \pm 0.35$ & AlNi20at\% ${ }^{[25]}$ & $105 \pm 5$ & $375 \pm 12$ \\
1498 & & $3.97 \pm 0.40$ & & & \\
\hline
\end{tabular}

Table 1 Temperature dependent self-diffusion coefficients of Ag and AlAg20.4at\% (left part) as well as the activation energy $E_{A}$ and prefactor $D_{0}$ of the Arrhenius equation (right part) for the systems presented here and systems previously reported in literature. Values shown in italic were taken from literature references.

tabulated for the liquid state [1]. For AlAg20.4at\% the thermodynamic factor at 983K is calculated to 0.69 . In order to calculate $D_{I D}$ in the absence of self-diffusion coefficients of $\mathrm{Al}$ for this alloy it is assumed that the self-diffusion coefficients of $\mathrm{Al}$ and of $\mathrm{Ag}$ are in the liquid roughly equal. Using the QENS determined Ag self-diffusion coefficient of $5.5 \pm 0.6 \times 10^{-9} \mathrm{~m}^{2} \mathrm{~s}^{-1}$ the Darken interdiffusion coefficient is calculated to $3.8 \times 10^{-9} \mathrm{~m}^{2} \mathrm{~s}^{-1}$. Assuming a $20 \%$ error on the Al self-diffusion coefficient and a $10 \%$ error on the thermodynamic factor the error bar of the Darken interdiffusion coefficient is about $0.4 \times 10^{-9} \mathrm{~m}^{2} \mathrm{~s}^{-1}$. Hence, the Darken interdiffusion coefficient is equal within error bars to the measured interdiffusion coefficient of $4.1 \pm 0.4 \times 10^{-9} \mathrm{~m}^{2} \mathrm{~s}^{-1}$. This means that for AlAg20.4at\% the Darken equation appears to be valid. Cross-correlations appear not to be important. However, to generalize this statement further temperature and concentration dependent measurements are required on the Al-Ag system.

Interesting to note is also that for the lowest measured Ag concentration (2at\%), the interdiffusion coefficient appears to be enhanced compared with the self-diffusion coefficient of pure liquid Al taken from Ref. [22]. Considering again the Darken equation and a thermodynamic factor that is approaching unity from below, this strongly indicates that Ag self-diffusion is faster than $\mathrm{Al}$ self-diffusion at these low concentrations. This is quite different to observations made for $\mathrm{Al}-\mathrm{Ni}$ [26] and $\mathrm{Al}-\mathrm{Cu}$ [30] where the solute diffusion even at low solute concentrations was lower than the diffusion of the pure solvent $\mathrm{Al}$.

Fig. 5 (right) depicts temperature dependent self-diffusion coefficients of the pure metals $\mathrm{Al}$ [23], $\mathrm{Cu}$ [28], Ni [29] and our data for $\mathrm{Ag}$ as well as solute self-diffusion coefficients for AlCu20at\% [9], AlNi20at\% [25] and our data for AlAg20.4at\% alloys. All temperature dependencies show Arrhenius behavior over the investigated temperature range. The diffusion coefficients of Ag and AlAg20.4at\% melts as well as the activation energies $E_{A}$ and $D_{0}$ values of all melts are listed in Table 1. Compared with the Al-Cu and Al-Ni system, Ag self-diffusion close to the melting temperature is faster than $\mathrm{Cu}$ and $\mathrm{Ni}$ self-diffusion in the $\mathrm{Al}-\mathrm{Cu}$ and $\mathrm{Al}-\mathrm{Ni}$ alloys of the same composition. AlAg20.4at\% has about a factor of two lower activation energy for solute selfdiffusion compared with the other alloy systems. Its $\mathrm{D}_{0}$ value is about one half to one third the value of AlNi20at\% and AlCu20at\%, respectively. For the pure liquids Ag shows equally low activation energy as $\mathrm{Al}$. Its $\mathrm{D}_{0}$ value is the lowest of the here reported alloy systems.

\section{Conclusion}

In the present paper the benefit was shown of combining the long-capillary method with X-ray radiography for in situ observation of the diffusion process in binary metal alloy melts with the aim of an accurate chemical diffusion coefficient determination. Interdiffusion coefficients for Al-Ag up to $42.5 \mathrm{at} \% \mathrm{Ag}$ were reported at a single temperature of $983 \mathrm{~K}$. The interdiffusion coefficients show 
first a decrease with increasing Ag content up to 20at\% Ag-content and further remain nearly constant. At low Ag content ( $<2 \mathrm{at} \% \mathrm{Ag}$ ) the interdiffusion coefficient appears to be larger than the self-diffusion coefficient of Al.

Ag self-diffusion was further measured using QENS for AlAg20.4at\% and pure liquid Ag. The Ag self-diffusion coefficient decreases with increasing Ag-content. A comparison of self-diffusion and interdiffusion coefficients shows that the interdiffusion coefficient at $20.4 \mathrm{at} \% \mathrm{Ag}$ is smaller than the Ag self-diffusion coefficient at equal composition. Considering thermodynamic driving forces, it is shown that the Darken relation is valid for this alloy.

\section{Acknowledgments}

We thank our colleagues Thomas Voigtmann and Georg Lohöfer for carefully reading the manuscript. We thank our colleague Matthias Kolbe for supporting the post-mortem analysis of the solidified Al-Cu diffusion samples by EDX analysis using the Institute's scanning electron microscopy station. We gratefully acknowledge Dr.-Ing. Wilfried Behr at Forschungszentrum Jülich GmbH, Jülich, Germany, for electron beam welding of the neutron sample holders. The Heinz Maier-Leibnitz Zentrum (MLZ), Garching, Germany, is gratefully acknowledged for the beamtime on their time-of-flight spectrometer TOFTOF.

\section{References}

[1] V. T. Witusiewicz, U. Hecht, S. G. Fries and S. Rex, J. Alloys Comp. Vol. 385 (2004), p. 133

[2] V. T. Witusiewicz, U. Hecht, S. G. Fries, S. Rex, J. Alloys Comp. Vol. 385 (2004), p. 217

[3] A. Genau and L. Ratke, Int. J. Mat. Res. Vol. 103 (2012), p. 469

[4] A. Dennstedt and L. Ratke, Trans. Ind. Inst. Metals Vol. 65 (2012), p. 777

[5] A. Dennstedt, L. Ratke, A. Choudhury and B. Nestler, Metall. Microstruct. Anal. Vol. 2 (2013), p. 140

[6] J. Hotzer, M. Jainta, P. Steinmetz, B. Nestler, A. Dennstedt, A. Genau, M. Bauer, H. Koestler and U. Rude, Acta Mater. Vol 93 (2015), p. 194

[7] Y. Du, Y. A. Chang, B. Huang, W. Gong, Z. Jin, H. Xu, Z. Yuan, Y. Liu, Y. He and F.-Y. Xie, Mat. Sci. Eng. A Vol. 363 (2003), p. 140

[8] Y. Du, L. J. Zhang, S. L. Cui, D. D. Zhao, D. D. Liu, W. B. Zhang, W. H. Sun and W. Q. Jie, Sci. China-Technol. Sci. Vol. 55 (2012), p. 306

[9] B. Zhang, A. Griesche and A. Meyer, Phys. Rev. Lett. Vol. 104 (2010), 035902

[10]F. Kargl, M. Engelhardt, F. Yang, P. Schmakat, B. Schillinger, A. Griesche and A. Meyer, J. Phys.: Condens. Matter Vol. 23 (2011), 254201

[11] F. Kargl, E. Sondermann, H. Weis, and A. Meyer, High Temp.-High Press. Vol. 42 (2013), p. 3

[12] A. Griesche, B. Zhang, E. Solórzano, and F. Garcia-Moreno, Rev. Sci. Instrum. Methods Vol. 81 (2010), 056104

[13] A. Meyer, EPJ Web of Conf. Vol. 83 (2015), 01002

[14] G.G. Simeoni, R. G. Valicu, G. Borchert, P. Böni, N. G. Rasmussen, F. Yang, F. Kordel, D. Holland-Moritz, F. Kargl and A. Meyer, Appl. Phys. Lett. (accepted)

[15] M. Engelhardt, Diffusion of mass in liquid AlCuAg and the binary subsystems, $\mathrm{PhD}$ thesis RWTH Aachen University (2014)

[16] A. Bruson and M. Gerl, Phys. Rev. B Vol. 21 (1980), p. 5447 
[17] G. Mathiak, A. Griesche, K. H. Kraatz and G. Frohberg, J. Non-Cryst. Solids Vol. 205 (1996), p. 412

[18] A. Griesche, M.-P. Macht and G. Frohberg, J. Non-Cryst. Solids Vol. 353 (2007), p. 3305

[19] R. M. Banish and L. B. Jalbert, Adv. Space Res. Vol. 24 (1999), p. 1311

[20] B. A. Beer, Annalen der Physik Vol. 86 (1952), p. 78

[21] A. Griesche, F. Garcia-Moreno, M. Macht, and G. Frohberg, Mat. Sci. Forum Vol. 508 (2006), p. 567

[22] H. Weis, Struktur und Dynamik in flüssigem Germanium und Silizium-Germanium, PhD thesis, Ruhr Universität Bochum (2012)

[23] F. Kargl, H. Weis, T. Unruh, and A. Meyer J. Phys.: Conf. Series Vol. 340 (2012), 012077

[24] S. K. Das, J. Horbach, M. M. Koza, S. Mavila Chatoth, and A. Meyer, Appl. Phys. Lett. Vol. 86 (2005), 011918.

[25] J. Horbach, S. K. Das, A. Griesche, M.-P. Macht, G. Frohberg, and A. Meyer, Phys. Rev. B Vol. 75 (2007), 174304

[26] L. S. Darken, Trans. AIME Vol. 175 (1948), p. 184

[27] E. Sondermann, F. Kargl, T. Unruh, and A. Meyer, submitted to Phys. Rev. Lett. (2015)

[28] A. Meyer, Phys. Rev. B Vol. 81 (2010), 012102

[29] A. Meyer, S. Stüber, D. Holland-Moritz, O. Heinen, and T. Unruh, Phys. Rev. B Vol. 77 (2008), 092201

[30] F. Kargl et al. (in preparation) 